\title{
ON THE MINKOWSKI MEASURABILITY OF FRACTALS
}

\author{
K. J. FALCONER
}

(Communicated by Andrew M. Bruckner)

\begin{abstract}
This note addresses two aspects of Minkowski measurability. First we present a short "dynamical systems" proof of the characterization of Minkowski measurable compact subsets of $\mathbb{R}$. Second, we use a renewal theory argument to point out that "most" self-similar fractals are Minkowski measurt able and calculate their Minkowski content.
\end{abstract}

\section{INTRODUCTION}

Let $F_{\varepsilon}$ denote the $\varepsilon$-neighbourhood of a set $F \subseteq \mathbb{R}^{n}$, i.e.,

$$
F_{\varepsilon}=\left\{x \in \mathbb{R}^{n}: \operatorname{dist}(x, F) \leq \varepsilon\right\} .
$$

We study the behaviour of $V\left(F_{\varepsilon}\right)$ as $\varepsilon$ approaches 0 , where $V$ denotes $n$ dimensional volume (Lebesgue measure). This may be used to define the Minkowski dimension, equivalently the box-counting dimension, of $F$, see [ 3 , $\S 3.1]$. In particular, if $V\left(F_{\varepsilon}\right) \approx \varepsilon^{n-d}$ as $\varepsilon \rightarrow 0$, then the Minkowski dimension or box-counting dimension of $F$ equals $d$ (this is the situation that we are particularly interested in here). In the nicest case, $V\left(F_{\varepsilon}\right) \sim c \varepsilon^{n-d}$ for some $c$ $(0<c<\infty)$, in which case we say that $F$ is $d$-dimensional Minkowski measurable, with Minkowski content $c$. (Note that $f(t) \approx g(t)$ as $t \rightarrow 0$ means there exist positive constants $a, b$ such that $a f(t) \leq g(t) \leq b f(t)$ for all sufficiently small $t$ and that $f(t) \sim g(t)$ means $f(t) / g(t) \rightarrow 1$ as $t \rightarrow 0$. We use a similar notation for $t \rightarrow \infty$.) Smooth or rectifiable curves are 1-dimensional Minkowski measurable, smooth surfaces are 2-dimensional Minkowski measurable, and so on (see Federer [4, 3.2.29]). Here we investigate conditions for fractal sets to be Minkowski measurable.

It is possible to give a complete characterization of Minkowski measurable compact subsets of $\mathbb{R}$. This was done by Lapidus and Pomerance $[12,13]$ in their treatment of the 1-dimensional Weyl-Berry conjecture. The lengths of the complementary intervals to $F$ are crucial. Let $I$ be a bounded closed interval, with $I_{n}$ disjoint open subintervals of $I$ satisfying $\left|I_{n}\right| \geq\left|I_{n+1}\right| \quad(n=1,2, \ldots)$ and $|I|=\sum_{n=1}^{\infty}\left|I_{n}\right| . \quad(|J|$ denotes the length of the interval $J$.$) Then the$ compact set $F=I \backslash \bigcup_{n=1}^{\infty} I_{n}$ is $d$-dimensional Minkowski measurable if and

Received by the editors September 15, 1992 and, in revised form, November 11, 1992 and July $1,1993$.

1991 Mathematics Subject Classification. Primary 28A80; Secondary 26A12, 60K10. 
only if $\left|I_{n}\right| \sim c n^{-1 / d}$ as $n \rightarrow \infty$ for some $c>0$. This follows from a result on the asymptotic behaviour of certain sequences which was proved by Lapidus and Pomerance [13]. In $\S 2$ we give a shorter dynamical systems proof of this, leading to a concise derivation of this characterization of Minkowski measurable subsets of $\mathbb{R}$. This characterization is of particular relevance to the work by Lapidus and Pomerance on the 1-dimensional version of the problem that is succinctly expressed as "Can you hear the dimension of a fractal?". We say a little more about this in $\S 4$.

In $\S 3$ we specialise to self-similar subsets of $\mathbb{R}$, showing that they are "almost always" Minkowski measurable and calculating their Minkowski content. This is in contrast to "exceptional" cases which happen to include the usual middlethird Cantor set, shown by Lapidus and Pomerance [13] not to be Minkowski measurable. These results follow easily using the renewal theory methods developed by Lalley [5-7].

The referee has pointed out that results similar to those of $\S 3$ have been obtained independently by Lapidus [10] and Kigami and Lapidus [8] in very recent work.

The author thanks Professor C. Pomerance for helpful comments on an earlier version of this paper.

\section{Characterization of Minkowski measurable subsets of $\mathbb{R}$}

In this section we give a rather shorter proof of the characterization of Minkowski measurable compact subsets of $\mathbb{R}$ originally given by Lapidus and Pomerance [13]. As there, we deduce the characterization from results on the asymptotic behaviour of sequences, which are stated in Corollary 2 below. The following proposition, which we prove using a dynamical systems argument, is a continuous version of the asymptotic result-an analogous proof using a discrete dynamical system would lead to the corollary directly, but the algebra is less straightforward.

Proposition 1. Let $f:[0, \infty) \rightarrow(0, \infty)$ be a nonincreasing continuous function with $\int_{0}^{\infty} f(t) d t<\infty$. Let $0<d<1$ and $\alpha, \beta>0$. Then as $t \rightarrow \infty$

$$
f(t) \approx t^{-1 / d}
$$

if and only if

$$
\alpha f(t)^{d-1} \int_{t}^{\infty} f(u) d u+\beta t f(t)^{d} \approx 1
$$

$$
f(t) \sim(\alpha d /(1-d)+\beta)^{-1 / d} t^{-1 / d}
$$

if and only if

$$
\alpha f(t)^{d-1} \int_{t}^{\infty} f(u) d u+\beta t f(t)^{d} \sim 1
$$

Proof. The implications (1) $\Rightarrow(2)$ and $(3) \Rightarrow(4)$ are easily verified by direct integration and substitution.

To establish the converses, define $x:[0, \infty) \rightarrow(0, \infty)$ by

$$
x(t)=t^{1 / d} f(t)
$$




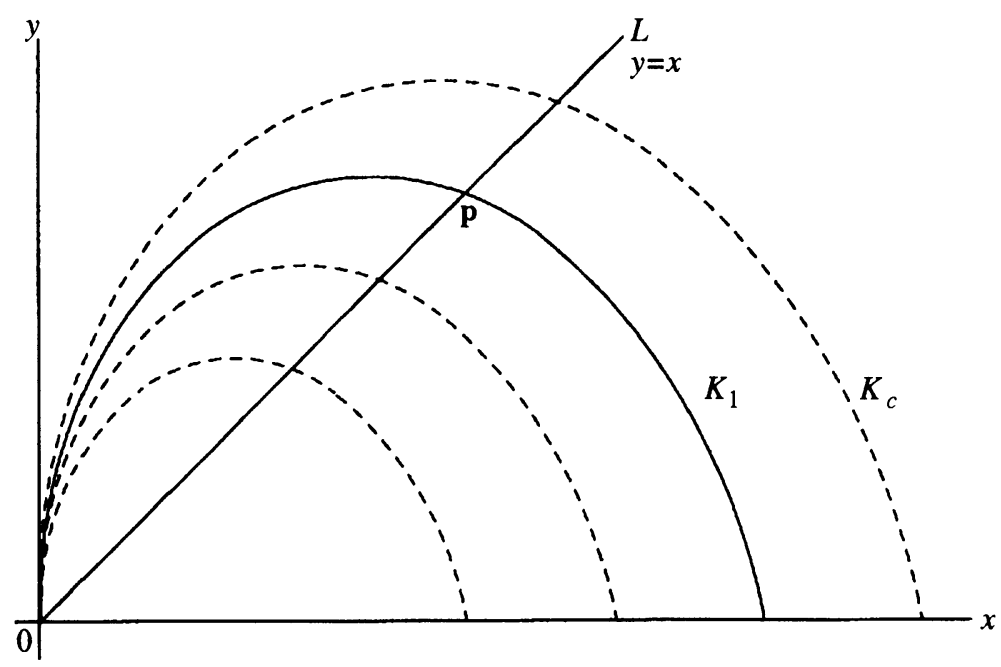

FigURE 1 . The family of curves $\left\{K_{c}\right\}$

and $y:[0, \infty) \rightarrow(0, \infty)$ by

$$
y(t)=((1-d) / d) t^{-1+1 / d} \int_{t}^{\infty} f(u) d u
$$

so that for $t>0$

$$
\begin{aligned}
y^{\prime}(t) & =((1-d) / d)^{2} t^{-2+1 / d} \int_{t}^{\infty} f(u) d u-((1-d) / d) t^{-1+1 / d} f(t) \\
& =((1-d) / d) t^{-1}[y(t)-x(t)] .
\end{aligned}
$$

We shall study $\mathbf{x}(t)=(x(t), y(t))$ as a dynamical system in $[0, \infty) \times[0, \infty)$.

For all $c>0$ let $K_{c}$ be the curve given by

$$
y=((1-d) / \alpha d)\left(c x^{1-d}-\beta x\right) \quad\left(0 \leq x \leq(c / \beta)^{1 / d}\right) .
$$

Thus $K_{c}$ is the set of points in $(0, \infty) \times[0, \infty)$ satisfying

$$
\alpha(d /(1-d)) x^{d-1} y+\beta x^{d}=c
$$

together with $(0,0)$. For each $c, K_{c}$ is a convex curve that approaches the origin tangentially to the $y$-axis and that meets the $x$-axis again at $\left((c / \beta)^{1 / d}, 0\right)$. The family of curves $\left\{K_{c}: 0<c<\infty\right\}$ varies continuously with $c$ in the obvious sense, with $K_{c^{\prime}}$ outside $K_{c}$ if $c<c^{\prime}$; see Figure 1 . We write $L$ for the line $y=x$, and $\mathbf{p}$ for the point of $K_{1} \cap L$ other than the origin.

Using (5) and (6), condition (2) becomes

$$
c_{1}<\alpha(d /(1-d)) x(t)^{d-1} y(t)+\beta x(t)^{d}<c_{2}
$$

for all $t$, for some $c_{1}, c_{2}$ with $0<c_{1} \leq c_{2}<\infty$. Thus, writing $R$ for the open region in $(0, \infty) \times(0, \infty)$ lying between $K_{c_{1}}$ and $K_{c_{2}}$, we have $(x(t), y(t)) \in R$ for all $t$. Since the curves $K_{c}$ approach the origin tangentially to the $y$ axis, the region $\left\{(x, y) \in R: 0<x \leq x_{0}\right\}$ lies above the line $L$ if $x_{0}$ $>0$ is chosen sufficiently small, so by equation (7) $y(t)$ is increasing whenever $x(t)<x_{0}$. Thus for $t>1$, the point $\mathbf{x}(t)$ can never enter the region 
$\left\{(x, y): x<x_{0}, y<\min \left(y(1), x_{0}\right)\right\}$; so $x(t)$ is bounded below. Trivially, $x(t)<\left(c_{2} / \beta\right)^{1 / d}$ for all $t$, which completes the proof of $(\mathrm{a})$.

Now suppose that the stronger condition (4) holds. Using (5) and (6) this condition becomes

$$
\alpha(d /(1-d)) x(t)^{d-1} y(t)+\beta x(t)^{d} \sim 1 .
$$

This means that $\mathbf{x}(t) \in K_{1+\varepsilon(t)}$ where $\varepsilon(t) \rightarrow 0$, so in particular

$$
\operatorname{dist}\left(\mathbf{x}(t), K_{1}\right) \rightarrow \quad \text { as } t \rightarrow \infty \text {. }
$$

We show that $\mathbf{x}(t) \rightarrow \mathbf{p}$ as $t \rightarrow \infty$ in two complementary cases.

Case (i). There exists $t_{0}>0$ such that $\mathbf{x}(t)$ does not strictly cross the line $L$ for any $t \geq t_{0}$. Thus $y(t)-x(t)$ does not change sign and $y(t)$ is monotonic for $t \geq t_{0}$, using (7). It follows that $y(t) \rightarrow y_{0}$ for some $y_{0} \in$ $[0, M]$, where $M=\sup \{y:(x, y) \in R\}$ and $R$ is as above. Since the set $\left\{x \in[0, \infty):\left(x, y_{0}\right) \in K_{1}\right\}$ is finite (in fact, contains at most two points), (9) implies that $x(t) \rightarrow x_{0}$ for some $x_{0}$ with $\left(x_{0}, y_{0}\right) \in K_{1}$. If $x_{0} \neq y_{0}$, then (7) implies that either $y^{\prime}(t) \geq a t^{-1}$ or $y^{\prime}(t) \leq-a t^{-1}$ for $t$ sufficiently large, where $a>0$, which would imply that $y(t) \rightarrow \infty$. Hence $\mathbf{x}(t) \rightarrow\left(y_{0}, y_{0}\right) \in K_{1}$; by part (a) $\mathbf{x}(t) \nrightarrow(0,0)$, so $\mathbf{x}(t) \rightarrow \mathbf{p}$.

Case (ii). The point $\mathbf{x}(t)$ lies strictly above $L$ and lies strictly below $L$ for arbitrary large $t$. Using (7), if $\mathbf{x}(t)$ is above $L$, then $y(t) \leq y\left(t_{+}\right)$where $t_{+}$ is the least number greater than $t$ such that $\mathbf{x}\left(t_{+}\right) \in L$; and if $\mathbf{x}(t)$ is below $L$, then $y(t) \leq y\left(t_{-}\right)$where $t_{-}$is the greatest number less than $t$ such that $\mathbf{x}\left(t_{-}\right) \in L$ (assuming that such a $t_{-}$exists, which is certainly the case for $t$ sufficiently large). Hence

$$
\limsup _{t \rightarrow \infty} y(t)=\lim _{t \rightarrow \infty}\{y(t):(x(t), y(t)) \in L\}
$$

and similarly

$$
\liminf _{t \rightarrow \infty} y(t)=\liminf _{t \rightarrow \infty}\{y(t):(x(t), y(t)) \in L\} .
$$

But if $\left\{t_{i}\right\}$ is any sequence with $t_{i} \rightarrow \infty$ and $\mathbf{x}\left(t_{i}\right) \in L$, (9) implies that $\mathbf{x}\left(t_{i}\right) \rightarrow \mathbf{p}$ (the sequence $\mathbf{x}\left(t_{i}\right)$ is bounded away from $(0,0)$ by part $(\mathbf{a})$ ). It follows from (10) and (11) that $y(t) \rightarrow y_{0}$ as $t \rightarrow \infty$, where $\mathbf{p}=\left(y_{0}, y_{0}\right)$. Using (9) again gives that $x(t) \rightarrow x_{0}$, where $\left(x_{0}, y_{0}\right) \in K_{1}$. Since $x_{0} \neq y_{0}$ would contradict that $\mathbf{x}(t)$ crosses $L$ for arbitrarily large $t$, we again conclude that $\left(x_{0}, y_{0}\right)=\mathbf{p}$.

Thus in both Cases (i) and (ii), $\mathbf{x}(t) \rightarrow \mathbf{p}=\left(x_{0}, x_{0}\right)$ as $t \rightarrow \infty$. Substituting in (8) with $c=1$ we get

$$
x(t) \rightarrow x_{0}=[\beta+\alpha d /(1-d)]^{-1},
$$

so (3) follows, using (5).

We apply Proposition 1 to obtain the characterization of Minkowski measurability given by Lapidus and Pomerance [13].

Proposition 2. Let I be a bounded closed interval in $\mathbb{R}$, and let $\left\{I_{n}\right\}_{n=1}^{\infty}$ be a sequence of disjoint open subintervals of $I$ with $\left|I_{n}\right| \geq\left|I_{n+1}\right|$ and $|I|=\sum_{n=1}^{\infty}\left|I_{n}\right|$. Define the compact set $F=I \backslash \bigcup_{n=1}^{\infty} I_{n}$. For all $d(0<d<1)$ and $c>0$ 
(a)

$$
\left|I_{n}\right| \approx n^{-1 / d} \quad \text { as } n \rightarrow \infty
$$

if and only if

$$
V\left(F_{\varepsilon}\right) \approx \varepsilon^{1-d} \quad \text { as } \varepsilon \rightarrow 0
$$

(b)

$$
\left|I_{n}\right| \sim 2^{1-1 / d} c^{1 / d}(1-d)^{1 / d} n^{-1 / d} \quad \text { as } n \rightarrow \infty
$$

if and only if

$$
V\left(F_{\varepsilon}\right) \sim c \varepsilon^{1-d} \quad \text { as } \varepsilon \rightarrow 0 .
$$

Proof. The $\varepsilon$-neighborhood $F_{\varepsilon}$ consists of $F$, all those intervals $I_{n}$ with $\left|I_{n}\right| \leq$ $2 \varepsilon$, two subintervals of lengths $\varepsilon$ inside every interval $I_{n}$ with $\left|I_{n}\right|>\varepsilon$, and an interval of length $\varepsilon$ at each end of $F$.

Hence, if $\varepsilon$ satisfies $\frac{1}{2}\left|I_{n+1}\right| \leq \varepsilon \leq \frac{1}{2}\left|I_{n}\right|$ (at least one such $n$ exists if $0<$ $\left.\varepsilon<\frac{1}{2}\left|I_{i}\right|\right)$, we have

$$
V\left(F_{\varepsilon}\right)=\sum_{i=n+1}^{\infty}\left|I_{i}\right|+2 n \varepsilon+2 \varepsilon .
$$

Direct substitution of (12) and (14) now gives (13) and (15) respectively (noting that $\left.\sum_{i=n+1}^{\infty} i^{-1 / d} \sim n^{1-1 / d} d /(1-d)\right)$.

For the converses, we have from (16) that

$$
\varepsilon^{d-1} V\left(F_{\varepsilon}\right)=\varepsilon^{d-1} \sum_{i=n+1}^{\infty}\left|I_{i}\right|+(2 n+2) \varepsilon^{d}
$$

Define $f:(1, \infty) \rightarrow(0, \infty)$ by

$$
f(t)=\frac{1}{2}(t-n)\left|I_{n+1}\right|+\frac{1}{2}(n+1-t)\left|I_{n}\right|
$$

where $n \leq t \leq n+1$. Then $f$ is continuous and nonincreasing with $f(t) \rightarrow 0$ as $t \rightarrow \infty$ and with $f(n)=\frac{1}{2}\left|I_{n}\right|$. Taking $\varepsilon=f(t)$ in (17) we get

$$
f(t)^{d-1} V\left(F_{f(t)}\right)=f(t)^{d-1} \sum_{i=n+1}^{\infty} 2 f(i)+(2 n+2) f(t)^{d}
$$

where $n \leq t \leq n+1$, so

$$
f(t)^{d-1} V\left(F_{f(t)}\right)=2 f(t)^{d-1} \int_{t}^{\infty} f(u) d u+2 t f(t)^{d}+O\left(f(t)^{d}\right) .
$$

(Notice that $\int_{t}^{\infty} f(u) d u=\sum_{i=n+1}^{\infty} f(i)+O(f(t))$ by an "integral test" estimate.)

Given (13), it follows from (18) that $f(t)=O\left(t^{-1 / d}\right)$ and thus that (2) holds with $\alpha=\beta=2$. It follows using Proposition $1(\mathrm{a})$ that $\left|I_{n}\right|=2 f(n) \approx n^{-1 / d}$ as $n \rightarrow \infty$.

Given (15), we get from (18) that (4) holds with $\alpha=\beta=2 / c$. By Proposition 1 (a) we get (3), which reduces to (14). 
It follows from Proposition 2(b) that $F$ is $d$-dimensional Minkowski measurable if and only if $\left|I_{n}\right| \sim a n^{-1 / d}$ for some $a>0$

\section{SELF-SIMILAR SETS}

We now specialize to self-similar subsets of $\mathbb{R}$ which generalise the middlethird Cantor set construction. The results given below are very much in the spirit of Lalley's work [5-7].

Let $I$ be a closed subinterval of $\mathbb{R}$, and let $\phi_{1}, \ldots, \phi_{m}: I \rightarrow I$ be contractions such that $\phi_{i}(I) \cap \phi_{j}(I)=\varnothing \quad(i \neq j)$. It is well known [3, $\left.\S 9.1\right]$ that there exists a unique, nonempty compact set $F$, called invariant set for $\left\{\phi_{1}, \ldots, \phi_{m}\right\}$, such that $F=\bigcup_{i=1}^{m} \phi_{i}(F)$. Write $F_{k}=\bigcup_{i_{1}, \ldots, i_{k}} \phi_{i_{1}} \circ \cdots \circ \phi_{i_{k}}(I)$ where the union is over all sequences $\left(i_{1}, \ldots, i_{k}\right)$ with $1 \leq i_{j} \leq m(1 \leq j \leq k)$. Then $F=\bigcap_{k=1}^{\infty} F_{k}$.

Suppose, for the moment, that $\phi_{i}$ are similarity transformations, i.e., $\left|\phi_{i}(x)-\phi_{j}(y)\right|=c_{i}|x-y| \quad(x, y \in I)$ where $0<c_{i}<1$; then $F$ is called a self-similar set. (The simplest example is the middle-third Cantor set, obtained by taking $\phi_{1}(x)=\frac{1}{3} x$ and $\phi_{2}(x)=\frac{1}{3} x+\frac{2}{3}$.) The box-counting dimension (and also the Hausdorff dimension) of $F$ is given by the unique positive $d$ satisfying $\sum_{i=1}^{k} c_{i}^{d}=1$. By choosing $I$ and ordering the $\phi_{i}$ appropriately, we may assume that $F$ has been constructed with $\phi_{1}(I), \ldots, \phi_{m}(I)$ subintervals of $I$ occurring in that order and with $\phi_{1}(I)$ and $\phi_{m}(I)$ each having an end in common with one of the ends of $I$. For convenience we assume that $F$ has been scaled so that $I=[0,1]$. We call $c_{1}, \ldots, c_{m}$ the ratios and $b_{1}, \ldots, b_{m-1}$ the gaps in the construction of $F$, where $b_{i}$ is the distance between $\phi_{i}(I)$ and $\phi_{i+1}(I) \quad(1 \leq i \leq m-1)$.

The following version of the renewal theorem is most convenient for our purposes. (The observation that (19) is equivalent to $N(t)=\sum_{i=1}^{m} N\left(t-r_{i}\right)+$ $\chi_{[0, \infty]}(t) \quad(t \geq 0), N(t)=0 \quad(t<0)$, followed by the substitution $Z(t)=$ $e^{-s t} N(t)$ reduces it to a more usual form of the renewal theorem.) We write $\operatorname{gp}\left\{r_{1}, \ldots, r_{m}\right\}$ for the additive subgroup of $\mathbb{R}$ generated by $\left\{r_{1}, \ldots, r_{m}\right\}$.

Proposition 3. Let $r_{i}>0 \quad(1 \leq i \leq m)$, and let

$$
N(t)=\#\left\{\left(i_{1}, \ldots, i_{k}\right): k \geq 0,1 \leq i_{j}<m \text {, and } \sum_{j=1}^{k} r_{i_{j}} \leq t\right\} \text {. }
$$

Let $s>0$ satisfy $\sum_{i=1}^{m} e^{-s r_{i}}=1$.

(a) If $\operatorname{gp}\left\{r_{1}, \ldots, r_{m}\right\}$ is dense in $\mathbb{R}$, then as $t \rightarrow \infty$

$$
N(t) \sim e^{s t} / s \sum r_{i} e^{-s r_{i}} .
$$

(b) If $\operatorname{gp}\left\{r_{1}, \ldots, r_{m}\right\}=h \mathbb{Z}$ where $h>0$, then as $t \rightarrow \infty$

$$
N(t) \sim e^{s t} g(t)
$$

where $g$ is a positive continuous function with period $h$.

Proof. See, for example, [7].

The following application of the renewal theorem is similar to that of Lalley [6]. Expression (20) gives the Minkowski content of a self-similar set in the "generic" case. 
Proposition 4. Let $F \subseteq \mathbb{R}$ be a self-similar set as described above, with ratios $c_{1}, \ldots, c_{m}$ and gaps $b_{1}, \ldots, b_{m-1}$. Let $d$ be the Minkowski dimension (or box-counting dimension) of $F$, so that $\sum_{i=1}^{m} c_{i}^{d}=1$.

(a) If $\operatorname{gp}\left\{\log c_{1}^{-1}, \ldots, \log c_{m}^{-1}\right\}$ is dense in $\mathbb{R}$, then as $\varepsilon \rightarrow 0$

$$
V\left(F_{\varepsilon}\right) \sim \varepsilon^{1-d} 2^{1-d}(1-d)^{-1}\left[\sum_{j=1}^{m-1} b_{j}^{-d} / d \sum_{i=1}^{m} c_{i}^{d} \log c_{i}^{-1}\right]
$$

in particular, $F$ is Minkowski measurable.

(b) If $\operatorname{gp}\left\{\log c_{1}^{-1}, \ldots, \log c_{n}^{-1}\right\}=h \mathbb{Z}$ for $h>0$, then as $\varepsilon \rightarrow 0$

$$
V\left(F_{\varepsilon}\right) \approx \varepsilon^{1-d} \text {. }
$$

Proof. For $u>0$ write

$$
\begin{aligned}
N(u) & =\#\left\{\text { sequences }\left(i_{1}, \ldots, i_{k}\right): k \geq 0,1 \leq i, j \leq m, \text { and } c_{i_{1}} c_{i_{2}} \cdots c_{i_{k}} \geq u\right\} \\
& =\#\left\{\text { sequences }\left(i_{1}, \ldots, i_{k}\right): \sum_{j=1}^{k} \log c_{i_{j}}^{-1} \leq-\log u\right\} .
\end{aligned}
$$

For case (a), we may apply Proposition 3(a) to deduce that

$$
N(u) \sim u^{-d} / d \sum_{i=1}^{m} c_{i}^{d} \log c_{i}^{-1}
$$

as $t \rightarrow \infty$. The $j$ th gap in $\phi_{i_{1}} \circ \cdots \circ \phi_{i_{k}}\left(\bigcup_{i=1}^{m} \phi_{i}(I)\right)$ has length $b_{j} c_{i_{1}} \cdots c_{i_{k}}$. Hence, if $\left\{I_{n}\right\}_{n=1}^{\infty}$ are the open intervals in the complement of $F$ arranged in decreasing order of length, we have for $w>0$,

$$
\begin{aligned}
\#\left\{n:\left|I_{n}\right| \geq w\right\} & =\sum_{j=1}^{m-1} \#\left\{\left(i_{1}, \ldots, i_{k}\right): b_{j} c_{i_{1}} c_{i_{2}} \cdots c_{i_{k}} \geq w\right\} \\
& =\sum_{j=1}^{m-1} N\left(w / b_{j}\right) \\
& \sim w^{-d} \sum_{j=1}^{m-1} b_{j}^{d} / d \sum_{i=1}^{m} c_{i}^{d} \log c_{i}^{-1} .
\end{aligned}
$$

Using (22) it follows that

$$
\left|I_{n}\right| \sim\left[\sum_{j=1}^{m-1} b_{j}^{d} / d \sum_{i=1}^{m} c_{i}^{d} \log c_{i}^{-1}\right]^{1 / d} n^{-1 / d},
$$

and Proposition 2(b) gives (20).

Case (b) follows in exactly the same way, but using Proposition 3(b) and 2(a).

We remark that these results extend to the much more general situation of "non linear" Cantor sets. Suppose now that $\phi_{1}, \ldots, \phi_{n}: I \rightarrow I$ are now conformal $C^{2}$ contractions where $I=\overline{\operatorname{int} I}$ is a subset of $\mathbb{R}^{n}$. Let $F$ be the invariant set of $\phi_{1}, \ldots, \phi_{m}$ (which exists in this situation), and assume that 
$\phi_{1}(F), \ldots, \phi_{m}(F)$ are disjoint. The Minkowski dimension of the invariant set $F$ is given by the unique positive number $d$ for which there exist $a, b>0$ such that

$$
a \leq \sum_{i_{1}, \ldots, i_{k}}\left|\left(\phi_{i_{1}} \circ \cdots \circ \phi_{i_{k}}\right)^{\prime}(x)\right|^{d} \leq b
$$

for all $k \in \mathbb{Z}^{+}$and $x \in I$. (See [1, 4] for a discussion of the thermodynamic formalism leading to this result.) For $x \in \phi_{i}(F)$ write $f(x)$ for the unique $y \in F$ such that $\phi_{i}(y)=x$; thus, $\phi_{i}$ is the local inverse of $f$ and $f(F)=F$.

We say that two functions $g, h \in C(F)$ are cohomologous if there exists a function $\psi \in C(F)$ such that $g-h=\psi-\psi \circ f$.

The following result may be obtained via Lalley's generalization of the renewal theorem to the nonlinear situation.

Proposition 5. Let $F$ be the invariant set for $\phi_{1}, \ldots, \phi_{m}: I \rightarrow I$ as above. Then $F$ is Minkowski measurable providing that the function $\log \left|f^{\prime}(\cdot)\right| \in C(F)$ is not cohomologous to any function taking values in a discrete additive subgroup of $\mathbb{R}$.

Note on Proof. This may be proved in an almost identical way to Theorem 12 of Lalley [6], which deals with the asymptotic behaviour of alternative approximations that can be used in box dimension calculations of the limit set of Schottky groups. The proof is fairly involved and hangs on a generalization of the renewal theorem to the nonlinear situation. However, the only change required, other than minor notational alternations, is that $N(\varepsilon, K)$ must be taken as $V\left(K_{\varepsilon}\right)$ rather than as the minimum cardinality of an $\varepsilon$-covering of a set $K$.

\section{FINAL REMARKS}

The question of whether fractals are Minkowski measurable has attracted recent prominence in work related to the Weyl-Berry conjecture on the distribution of eigenvalues of the Laplacian on domains with fractal boundaries; see [2, 8-15]. Particularly relevant is the paper by Lapidus and Pomerance [13] which gives an analysis of the conjecture in the 1-dimensional case.

As in $\S 2$, we let $I$ be a closed bounded interval and let $\left\{I_{n}\right\}$ be a sequence of disjoint open subintervals such that $|I|=\sum_{n=1}^{\infty}\left|I_{n}\right|$. We write $\Omega=\bigcup_{n=1}^{\infty} I_{n}$ and $F=I \backslash \Omega$. We consider the eigenvalue problem

$$
\begin{gathered}
\frac{\partial^{2} u}{\partial x^{2}}=-\lambda u \quad \text { on } \Omega, \\
u=0 \quad \text { on } \partial \Omega .
\end{gathered}
$$

(We imagine a vibrating string stretched over the gaps $I_{n}$ in the fractal $F$.) It is clear that the problem (24), (25) has a nontrivial solution if and only if $\sqrt{\lambda}$ is a multiple of $\pi /\left|I_{n}\right|$ for some $n$. Hence, if $N(\lambda)$ denotes the number of eigenvalues less than or equal to $\lambda$, we have

$$
N(\lambda)=\sum_{n=1}^{\infty}\left\lfloor\pi^{-1} \lambda^{1 / 2}\left|I_{n}\right|\right\rfloor
$$

where \lfloor\rfloor denotes "integer part of". 
Weyl's classical result on the distribution of eigenvalues of the Laplacian on a domain $\Omega$ in $\mathbb{R}^{n}$ is that $N(\lambda) \sim c \operatorname{vol}_{n}(\Omega) \lambda^{n / 2}$. (This follows easily from (26) in the case $n=1$.) Berry [2] conjectured that the next term in the asymptotic expansion reflects the "fractal" dimension of the bounds of $\Omega$. Lapidus and Pomerance [13] obtained very precise formulation of this in the 1-dimensional case in terms of Minkowski measurability and Minkowski content.

Their argument depends on two asymptotic results. The first is Proposition 2 above. The second is that if $\left\{a_{n}\right\}$ is a decreasing sequence of positive numbers with $a_{n} \sim a n^{-1 / d}$, then

$$
\sum_{n=1}^{\infty}\left(t a_{n}-\left\lfloor t a_{n}\right\rfloor\right) \sim-\zeta(d) a^{d} t^{d} \quad \text { as } t \rightarrow \infty
$$

where $\zeta$ is the Riemann zeta function (note $\zeta(d)<0$ for $0<d<1$ ). This was proved [13, Theorem 4.2] by breaking the series of (27) into three parts and using estimates of an analytic number-theoretic flavour.

To complete the argument, note that if $F \subseteq \mathbb{R}$ is $d$-dimensional Minkowski measurable with Minkowski content $c$, then (14) holds; so by (26) and (27)

$$
N(\lambda)=\pi^{-1}|I| \lambda^{1 / 2}+\zeta(d) \pi^{-d} 2^{d-1}(1-d) c \lambda^{d / 2}+o\left(\lambda^{d / 2}\right) .
$$

This formula holds for self-similar sets of the form considered in Proposition $4(\mathrm{a})$, so $N(\lambda)$ may be estimated in terms of the ratios and gaps of the set, using (20), i.e.,

$$
N(\lambda)=\pi^{-1} \lambda^{1 / 2}+\zeta(d) \pi^{-d}\left[\sum_{j=1}^{m-1} b_{j}^{d} / d \sum_{i=1}^{m} c_{i}^{d} \log c_{i}^{-1}\right] \lambda^{d / 2}+o\left(\lambda^{d / 2}\right) .
$$

In the same way, if $F$ merely satisfies $V\left(F_{\varepsilon}\right) \approx \varepsilon^{1-d}$, we get

$$
b_{1} \lambda^{d / 2} \leq \pi^{-1} \lambda^{1 / 2}|I|-N(\lambda) \leq b_{2} \lambda^{d / 2}
$$

where the positive constants $b_{1}, b_{2}$ may, in principle, be estimated in terms of the implicit constants in $V\left(F_{\varepsilon}\right) \approx \varepsilon^{1-d}$.

The reader is referred to a very interesting paper by Lapidus and Maier [11], showing that a converse of this question (roughly, if a formula such as (28) holds, then $F$ is Minkowski measurable) is equivalent to the Riemann hypothesis.

The relationship between the eigenvalues of the Laplacian in a domain in $\mathbb{R}^{n}$ and the dimension of the boundary is studied in [9] in the case $n \geq 2$. There is not in general such a convenient formula as (26) for $N(\lambda)$ in terms of the geometry of the domain.

We remark that a related but simpler problem concerns packing intervals into $\Omega$ (as defined above). We write $P(\varepsilon)$ for the maximum number of disjoint open intervals of length $\varepsilon$ that may be placed inside $\Omega=\bigcup_{n=1}^{\infty} I_{n}$. Then

$$
P(\varepsilon)=\sum_{n=1}^{\infty}\left\lfloor\left|I_{n}\right| \varepsilon^{-1}\right\rfloor
$$

so a similar argument shows that if $F$ is $d$-dimensional Minkowski measurable with Minkowski content $c$, then as $\varepsilon \rightarrow \infty$

$$
P(\varepsilon)=\varepsilon^{-1}|I|+\zeta(d) 2^{d-1}(1-d) c \varepsilon^{-d}+o\left(\varepsilon^{d}\right) .
$$




\section{REFERENCES}

1. T. Bedford, Applications of dynamical systems theory to fractals-a study of cookie cutter Cantor sets, Fractal Analysis and Geometry, Kluwer Academic, Dordrecht, 1991.

2. M. V. Berry, Some geometric aspects of wave motion: wavefront dislocations, diffraction catastrophes, diffractals, Geometry of the Laplace Operator, Proc. Sympos. Pure Math., vol. 36, Amer. Math. Soc., Providence, RI, 1980, pp. 13-38.

3. K. J. Falconer, Fractal geometry-Mathematical foundations and applications, Wiley, Chichester, 1990.

4. H. Federer, Geometric measure theory, Springer-Verlag, Berlin, 1969.

5. S. P. Lalley, The packing and covering functions of some self-similar fractals, Indiana Math. J. 37 (1988), 699-710.

6. __ Renewal theorems in symbolic dynamics, with applications to geodesic flow, noneuclidean tessellations and their fractal limits, Acta Math. 163 (1989), 1-55.

7. __ Probabilistic methods in certain counting problems of ergodic theory, Ergodic Theory, Symbolic Dynamics and Hyperbolic Spaces, Oxford Univ. Press, Oxford, 1991, pp. 223-258.

8. J. Kigami and M. L. Lapidus, Weyl's problem for the spectral distribution of Laplacians on p.c.f. self-similar fractals, Comm. Math. Phys. 158 (1993), 93-125.

9. M. L. Lapidus, Fractal drum, inverse spectral problems for elliptic operators and a partial resolution of the Weyl-Berry conjecture, Trans. Amer. Math. Soc. 325 (1991), 465-529.

10. _ Vibrations of fractal drums, the Riemann hypothesis, waves in fractal media, and the Weyl-Berry conjecture, Ordinary and Partial Differential Equations IV, Longman Sci. Tech., Essex, 1993, pp. 126-209.

11. M. L. Lapidus and H. Maier, The Riemann hypothesis, inverse spectral problem for vibrating fractal strings and the modified Weyl-Berry conjecture, J. London Math. Soc. (to appear).

12. M. L. Lapidus and C. Pomerance, Fonction zêta de Riemann et conjecture de Weyl-Berry par les tambours fractals, C. R. Acad. Sci. Paris Sér. I Math. 310 (1990), 343-348.

13. The Riemann zeta-function and the one-dimensional Weyl-Berry conjecture for fractal drums, Proc. London Math. Soc. (3) 66 (1993), 41-69.

14. D. Ruelle, Repellers for real analytic maps, Ergodic Theory Dynamical Systems 2 (1982), 99-109.

School of Mathematics, University of Walk, Bristol BS8 1TW, ENGLAND

Current address: Mathematical Institute, University of St. Andrews, Fife KY16 9SS, Scotland

E-mail address: kjfest-andrews.ac.uk 